\title{
Demokratische Transformation und Parteiverbote: Warum gibt es kein Parteiverbot in der südafrikanischen Verfassung?
}

\author{
Von Jörg Kemmerzell, Darmstadt*
}

\section{A. Einleitung ${ }^{1}$}

Das subsaharische Afrika wurde, ähnlich wie Osteuropa oder Teile Asiens, zwischen 1989 und 1994 von einer Welle der Demokratisierung ehemaliger Einparteienstaaten und Militärdiktaturen erfasst. ${ }^{2}$ Die Einführung bzw. Wiedereinführung von Mehrparteiensystemen war in vielen Staaten allerdings von Befürchtungen begleitet, dass die neue institutionelle Ordnung die Politisierung partikularer Identitäten möglicherweise prämiert und so zu einer Verschärfung gesellschaftlicher Konflikte führen könnte. Wenigstens 40 der 48 Staaten in der Region haben seit Beginn der neunziger Jahre gleichermaßen die rechtliche Möglichkeit von Parteiverboten geschaffen. ${ }^{3} 29$ Staaten erwähnen Parteiverbote explizit in der Verfassung, in den übrigen Fällen sind sie Gegenstand wenigstens der einfachen Gesetzgebung zum Parteien- oder Wahlrecht. Die meisten Verbotsnormen wollen einer Politisierung ethnischer und kultureller Identitäten (Ethnizität, Sprache, Abstammung, Religion) vorbeugen. Besonders deutlich wird dies am ruandischen Beispiel, wo mehrere Parteien unter dem Vorwurf, „divisionnisme“ zu schüren, verboten wurden. ${ }^{4}$ Zwar kann die Geltung rechtsstaatlicher Prinzipien unter dem derzeitigen ruandischen Regime sowie dessen demokratischer Charakter durchaus angezweifelt werden ${ }^{5}$, aber auch in Verfassungen vergleichsweise

Jörg Kemmerzell, wissenschaftlicher Mitarbeiter am Institut für Politikwissenschaft und im Exzellenzcluster „Die Herausbildung normativer Ordnungen“ an der Technischen Universität Darmstadt. E-mail: kemmerzell@pg.tu-darmstadt.de

Die Untersuchung beruht auf dem von der Fritz Thyssen Stiftung geförderten Forschungsprojekt „Managing Ethnic Conflict through Institutional Engineering. Ethnic Party Bans in Africa“. Die Fritz Thyssen Stiftung ermöglichte auch die Feldforschung in Südafrika.

Siehe Michael Bratton / Nicolas van de Walle, Democratic Experiments in Africa. Regime Transitions in Comparative Perspective, 1997.

Anika Becher, Gesellschaftliche Konfliktlinien und politische Gewalt - Zum Verbot ethnischer Parteien im subsaharischen Afrika, in: Karsten Grabow, / Patrick Köllner (Hrsg.): Parteien und ihre Wähler. Gesellschaftliche Konfliktlinien und Wählermobilisierung im internationalen Vergleich, 2007, S. 83-102.

Peter Niesen, Demokratieerhalt durch Parteiverbot? Das Fallbeispiel Ruanda, Zeitschrift für Menschenrechte 1 (2008), S. 64-90. Für eine systematische Analyse solcher anti-partikularistischer Parteiverbote, mit den Schwerpunkten Türkei und Israel, siehe Nancy Rosenblum, Banning Parties: Religious and Ethnic Partisanship in Multicultural Democracies, Journal of Law and Ethics of Human Rights 1 (2007), S. 17-75.

5

Philip Reyntiens, Rwanda, Ten Years on. From Genocide to Dictatorship, African Affairs 103 (2004), S. 177-210. 
demokratischer Staaten wie Ghana oder Benin findet sich das Instrument des Parteiverbotes. Es fällt zudem auf, dass die Parteiverbotsnormen offenbar nicht zum institutionellen Erbe des autoritären post-kolonialen Staates gehören, sondern eine neue Erscheinung in Transformationsverfassungen darstellen. ${ }^{6}$

Südafrika ist eines der wenigen afrikanischen Länder, die auf Parteiverbotsmöglichkeiten verzichtet haben. Dies ist nicht nur im Vergleich zur zumeist anders gearteten regionalen Praxis erklärungsbedürftig sondern auch, weil sich eine Reihe möglicher Gründe für die Wahl dieser verhältnismäßig ,repressiven“ Institution finden lassen. Zu nennen sind dabei die Erfahrung eines auf ethnischer Diskriminierung aufbauenden autoritären Regimes, die Spaltung der Gesellschaft entlang ethnischer Konfliktlinien zwischen „schwarz“ und „weiß“, aber auch innerhalb der schwarzen Mehrheitsbevölkerung, sowie die deutliche Präsenz „extremistischer“ Kräfte. Trotz dieser Faktoren mündete die Transition in Südafrika aber in eine ausgesprochen liberale, stark auf Grundrechten basierende Verfassung. Nach einer Einführung in das Konzept des Parteiverbots in Abschnitt B fragt Abschnitt C, ob im Zeitraum der demokratischen Transformation (1990 bis 1994) bestimmte Gründe eine „,militante“ Verfassungskonzeption ${ }^{7}$ in Südafrika hätten rechtfertigen können. Hierzu wird die politische Situation während dieses Transformationsprozesses beleuchtet. Abschnitt D widmet sich einschlägigen Verfassungsnormen, wie insbesondere „Freedom of Association“ (Art. 18), „Political Rights“ (Art. 19) und „Limitation of Rights“ (Art. 36). Abschnitt E skizziert einen mehrdimensionalen Erklärungsansatz für den Verzicht auf eine Parteiverbotsnorm. Den Abschluss bilden eine transformationstheoretische Erklärung des Sachverhaltes und ein Ausblick in komparatistischer Absicht.

\section{B. Das Konzept des Parteiverbotes}

In den Kategorien von Gregory Fox und Georg Nolte, den Verfassern der maßgeblichen Studie zum Verbot politischer Parteien, kann die südafrikanische Demokratie dem Typ der „tolerant substantive democracy“ zugeordnet werden. ${ }^{8}$ "Substantive“, weil der Verfassung eine Reihe von „founding provisions“ sowie ein ausgeprägter Katalog von Grundrechten zugrunde liegen; ,tolerant“, weil sie außer einer allgemeinen Grundrechts-Einschränkungsklausel keinen militanten Republikschutz vorsieht.

Fox und Nolte behandeln unter dem Titel „Intolerant Democracies“ sowohl nationale Traditionen der militanten Demokratie als auch internationale Rechtsnormen, die den Umgang mit antidemokratischen Akteuren, darunter insbesondere Parteien, betreffen. Ausge-

6

Matthias Basedau / Matthijs Bogaards / Christof Hartmann / Peter Niesen, (Hrsg), Ethnic Party Bans in Africa: A Research Agenda, German Law Journal 8 (2007).

7

Der Begriff „,militant democracy“ wurde von Karl Loewenstein (1937) geprägt, der angesichts der faschistischen Herausforderung im Europa der dreißiger Jahre einen militanten Standpunkt der Demokratien gegenüber ihren Feinden einforderte. Verbote faschistischer Parteien bilden ein Kernelement dieses Ansatzes.

Gregory H. Fox / Georg Nolte, Intolerant Democracies, Harvard Law Journal 36 (1995), S. 27. 
hend von einem liberal begründeten „Selbstabschaffungsverbot“ für Demokratien postulieren sie nicht nur ein „Rückfallverbot“ in vordemokratische Zustände, sondern plausibilisieren auch eine Verpflichtung, Maßnahmen des aktiven Demokratieschutzes zu ergreifen. Das Bekenntnis zur „Allgemeinen Erklärung der Menschenrechte“ oder der Beitritt zum „Internationalen Pakt über bürgerliche und politische Rechte“ verpflichtet Demokratien demnach zu Maßnahmen des Selbstschutzes, die auch bestimmte Wahlentscheidungen suspendieren können. Fox/Nolte erläutern das aktive Selbstschutzgebot im Vergleich zu einem schlichten Rückfallverbot anhand des Verbots der algerischen FIS im Jahr 1991: Nachdem die offen antidemokratische FIS die absolute Mehrheit bei den Parlamentswahlen gewonnen hatte, wurde sie von der noch amtierenden Regierung verboten. Eine passive Auslegung des Rückfallverbots hätte lediglich eine spätere FIS-Regierung verpflichtet, die demokratischen Institutionen aufrecht zu erhalten. Das Verbot der FIS sehen Fox/Nolte in Übereinstimmung mit dem von ihnen befürworteten aktiven Schutzgebot. Wenn die Regierungsübernahme durch eine bestimmte Partei absehbar zur dauerhaften Suspendierung bürgerlicher und politischer Rechte führt, ist das Verbot dieser Partei gerechtfertigt, auch wenn auf kurze Sicht politische Rechte dadurch verletzt werden. „Protecting future voting majorities“" wird zu einer auch völkerrechtlich verpflichtenden Aufgabe, derer sich eine Gesellschaft auch durch die Ausübung der Volkssouveränität nicht entledigen darf: „(...) there are certain things a society cannot choose to do to itself". 9

Die Überlegungen Fox und Noltes beziehen sich explizit auf stabile Demokratien. ${ }^{10}$ Auf sie angewendet, entspricht ihr Modell des Demokratieschutzes in etwa der ,wehrhaften Demokratie“, wie sie das Bundesverfassungsgericht in den Parteiverbotsurteilen gegen SRP und KPD in den 1950er Jahren entwickelt hat: Eine Partei kann verboten werden, wenn sie aktiv die freiheitlich demokratische Ordnung des Grundgesetzes bekämpft. Unerheblich ist hingegen, mit welchen Mitteln sie diese bekämpft und ob von ihr eine akute Gefahr ausgeht. Peter Niesen definiert eine solche Konzeption von Parteiverboten als ,,anti-extremistisch“. ${ }^{11}$ Charakteristisch für den Antiextremismus ist seine abstrakte Fassung, die nicht des Rückbezuges auf konkret erfahrenes Unrecht bedarf. Über stabile Demokratien hinausgehend erwähnen Fox und Nolte das besondere Risiko junger Demokratien, in vordemokratische Verhältnisse zurückzufallen. Die Einschränkung anti-demokratischer Parteien er-

In der Auswahl Algeriens, wenn auch nur zur Illustration, liegt indes eine Schwäche ihrer Analyse, da sicher in Frage steht, inwiefern Algerien 1991 wenigstens als formale Demokratie betrachtet werden kann. Es liegt die Vermutung nahe, dass es Fox und Nolte weniger um den Bestand demokratischer Prozeduren als um die Chance zur Verwirklichung von Menschenrechten geht.

11

Peter Niesen, Zwischen Pfadabhängigkeit und Kommensuration: Verbote politischer Parteien in Europa, in: Christian Joerges / Matthias Mahlmann / Ulrich K. Preuß (Hrsg.), „Schmerzliche Erfahrungen“ der Vergangenheit und der Prozess der Konstitutionalisierung Europas, Wiesbaden 2008, S. 258-273. 
scheint in ihnen als ein Komplement des ,right of revolution against undemocratic regimes (which) is at the core of traditional democratic theory“. ${ }^{12}$

Dieses Argument leitet über zu dem von Niesen so bezeichneten ,negativ-republikanischen“ Paradigma, in dem Parteiverbote als „Rückfallsperre“ und symbolische Abgrenzung gegenüber einem undemokratischen Vorgängerregime konzeptualisiert werden. ${ }^{13}$ Der negative Republikanismus gewinnt seine Relevanz nur als konkrete Negation eines historischen Regimes, als Vorbild dient das Verbot der Faschistischen Partei in der italienischen Verfassung von 1947.

\section{Mögliche Begründungen für Parteiverbote in Südafrika}

Die südafrikanische Verfassung kennt keine Verbotsnorm, obwohl während der Transformation bzw. in der Phase der Verfassungsgebung Gründe für beide Typen von Parteiverboten bestanden hätten: Parteiverbote können erstens als eine Form von Vergangenheitsaufarbeitung verstanden werden, die auf eine symbolische und faktische Abgrenzung vom alten (Unrechts-)Regime zielt. Ruti Teitel erwähnt das Parteiverbot am Beispiel der jungen Bundesrepublik im breiteren Kontext von „transitional justice“. ${ }^{14}$ Eine explizite Abgrenzung vom Vorgängerregime findet sich neben der italienischen auch in der portugiesischen Verfassung. Diese verbietet faschistische Organisationen mit der Begründung, die Republik beruhe auf der Überwindung des faschistischen Regimes. Auf Südafrika übertragen hätte dies nahegelegt, Parteien zu verbieten, die sich positiv zum Apartheidsystem stellen und dessen Wiedereinführung fordern. Die „Constitutional Guidelines“, die der African National Congress noch 1988 für eine Post-Apartheid-Ordnung formuliert hatte, betonten auch tatsächlich die Bindung der politischen Akteure an das offensiv formulierte Ziel, Diskriminierung auf Basis rassischer Zugehörigkeit entschlossen zu bekämpfen. ${ }^{15}$ Die „Guidelines“ des ANC sahen im Einzelnen die Möglichkeit vor, ,to outlaw the advocacy and practice of racism, fascism, nazism or tribalism, or the incitement of ethnic or regional exclusiveness or hatred“ (Guideline k). Zudem wurde der Staat verpflichtet „to eradicate apartheid in all its forms“ (Guideline i) und auf ein militantes Demokratieverständnis festgelegt: „All organs of government (...) shall be (...) dedicated to defending the principles of the constitution" (Guideline d).

Zweitens lässt sich Parteiverboten durch den Ausschluss historischen Unrechts eine freiheitsverbürgende Funktion zuschreiben. Hier kann auf die Forderung nach „liberatory intolerance“" als politischem Leitbild eines neuen Südafrika verwiesen werden, das vom

14 Ruti Teitel, Transitional Justice, Oxford 2000, S. 178. (http://www.disa.ukzn.ac.za/index.php?option=com_displaydc\&recordID=pam19890000.026.021 $.000)$. 
späteren Minister Pallo Jordan 1988 propagiert wurde. „Liberatory intolerance“ stellt in dieser Perspektive eine Antwort auf einen ,radically evil enemy“ dar: „Pallo Jordan noted 1988: 'apartheid is a doctrine that is radically evil'; therefore Jordan called for 'liberatory intolerance'. Through this theory, the liberal notion of tolerance is rejected since apartheid could not be viewed as just another competitor in the marketplace of ideas". 16 Die obigen Leitlinien des ANC, die einen Ausschluss von rassische oder ethnische Konfliktlinien mobilisierenden Parteien nahe legen könnten, finden in diesen Ausführungen ihre theoretische Grundlegung.

Im Rahmen der antiextremistischen Konzeption bewegen sich hingegen stabilitätsorientierte Argumente. Die „unsicheren“ politischen Verhältnisse während der Liberalisierungsphase (1990-1994), die manche Autoren gar von einem ,low-intensity civil war“ ${ }^{17}$ begleitet sehen, stellten den Erfolg der Transformation in Frage. ${ }^{18}$ In diesem Zusammenhang ist zum Beispiel an Giovanni Sartoris Betonung der stabilisierenden Wirkung der Parteiverbote für die junge Demokratie der Bundesrepublik zu erinnern. So hätte nicht in erster Linie die Fünf-Prozent-Sperrklausel eine Konzentration und De-Polarisierung des Parteiensystems bewirkt, sondern vor allem die Parteiverbote des Bundesverfassungsgerichts: „The reduction to just three of the parties of the Bonn Republic has instead a great deal to do with the fact that the Constitutional Court outlawed as anti-democratic, and therefore as unconstitutional, both the Communist and the Neo-Nazi parties“. ${ }^{19}$

Für den südafrikanischen Fall müssen in diesem Kontext die „COSAG Parties“ (Concerned South African Group) erwähnt werden, ein Zusammenschluss politischer Akteure, die im Juni 1993 die Teilnahme am „Multi Party Negotiation Process“ (MPNP) aufkündigten mit der Perspektive, weder die neue Verfassung zu akzeptieren, noch an den für 1994 geplanten Wahlen teilzunehmen. ${ }^{20}$ Diese sehr heterogene Gruppe ${ }^{21}$ wurde im Wesentlichen dadurch zusammengeführt, dass die Beteiligten in der engen Zusammenarbeit von Regierung (gestellt von der National Party) und African National Congress die Gefahr ihrer Marginalisierung sahen. ${ }^{22}$ Grundlage dieser Befürchtung war insbesondere der

James L. Gibson, Does truth lead to reconciliation? Testing the causal assumptions of the South African Truth and Reconciliation Process, 2002 (http://www.utexas.edu/law/news/colloquium/ papers/UTVersio.pdf).

Hennie Kotzé / Pierre du Toit, Historical Contexts, in: Ursula J. van Beek (ed.), Democracy under Construction. Patterns from four Continents, 2005, S. 264, besprochen in VRÜ 41 (2008), S. 587. Timothy D. Sisk, Democratization in South Africa. The Elusive Social Contract, Princeton 1995. Giovanni Sartori, Comparative Constitutional Engineering, New York 1994, S. 19.

Richard Spitz / Matthew Chaskalson, The Politics of Transition. A hidden History of South Africa's negotiated Settlemet, Oxford, S. 212.

21 Bestehend aus IFP (Inkatha Freedom Party), CP (Conservative Party), AVU (Afrikaner Volksunie), KwaZulu Government, Homeland Governments Of Bophuthatswana and Ciskei. möglich. Detaillierte Analysen der demokratischen Transformation Südafrikas und ihrer Vorgeschichte, denen auch dieser Text unerlässliche Basisinformationen verdankt, finden sich unter 
„Record of Understanding“, in dem die Regierung und der ANC eine enge Zusammenarbeit im Verfassungsgebungsprozess vereinbart und bereits eine Reihe inhaltlicher Festlegungen getroffen hatten. ${ }^{23}$ Die COSAG Parteien lehnten die politische und institutionelle Ausrichtung des Verhandlungsprozesses im Ganzen ab. Insbesondere befürchteten sie eine zu starke Unitarisierung des zukünftigen Staates, eine Unterminierung des Gewohnheitsrechts (,traditional law“) und eine dauerhafte Majorisierung der Weißen durch die schwarze Mehrheit. Zusammengefasst können folgende Kernanliegen der COSAG Parteien, die sich im Oktober 1993 als „Freedom Alliance““24 eine gemeinsame organisatorische Plattform gaben, identifiziert werden:

1. In Opposition gegen einen Einheitsstaat befürworteten sie eine föderale Staatsordnung, die explizit auf einem Bekenntnis zur regionalen Autonomie (,,acceptance of regionalism") beruht.

2. Die Anerkennung der regionalen Ebene als Träger staatlicher Macht.

3. Die Ablehnung des von der Regierung einseitig geführten Verhandlungs- und Friedensprozesses.

Zur problematischen Komposition dieser Allianz merken Richard Spitz und Matthew Chaskalson allerdings an ${ }^{25}$ :

„In practice, each party had its own interests. The Status of KwaZulu Natal, an Afrikaner Volkstaat, and the opposition of the Bophuthatswana and Ciskei homeland administrations in the new dispensation mattered at most to two COSAG parties at a given time. But substantive differences were counterbalanced by a common hatred of ANC-government bilateralism, as epitomised by sufficient consensus in decision-making, and by the hope that a united front of malcontents would compel the ANC and the government to reconsider the way forward".

Während sich die COSAG/Freedom Alliance Akteure offiziell zu einem friedlichen Prozess bekannten, verfolgten sie tatsächlich doch eine Doppelstrategie: „Proposals for the negotiating table were backed up by the threat of resistance if they were not accepted“. ${ }^{26}$ Dass Gewalt im Umkreis der COSAG Parteien auch aktiv angewendet wurde, zeigen zwei Ereignisse: Einmal die bürgerkriegsähnlichen Auseinandersetzungen zwischen Inkatha und ANC-Anhängern zwischen 1990 und 1994, die bis zu 15000 Menschenleben gekostet haben $^{27}$; zum anderen die Versuche weißer Extremisten, mit Gewalt den Regimewechsel

anderem bei Steven Friedman, The small Miracle. South Africa's negotiated Settlement, Johannesburg 1994, Timothy D. Sisk, oben Fn. 18, Allister Sparks, Morgen ist ein anderes Land, Berlin 1995 sowie Richard Spitz / Matthew Chaskalson, oben Fn. 20.

Hassen Ebrahim, The Soul of a Nation. Constitution-making in South Africa, Cape Town 1988, S. $588 \mathrm{ff.}$.

Ebrahim, oben Fn. 23, S. 230.

Spitz / Chaskalson, oben Fn. 20, S. 213.

26

Spitz, Chaskalson, oben Fn. 20, S. 232.

27

Es wäre historisch allerdings verfehlt, die Verantwortung für diese Ereignisse nur einer Seite zuzuschieben. Die Gewalt ging gleichermaßen von Inkatha und dem ANC aus und wurde zudem durch Provokationen des Geheimdienstes (,third force“) geschürt, wie der damalige Präsident de 
aufzuhalten und/oder wenigstens einen unabhängigen „Volksstaat“ zu errichten, in dem die Regeln der Apartheid weiter bestehen sollten. Hier ist zum einen der Überfall von AWBAnhängern auf die Kempton-Park-Verhandlungen (Juni 1993) zu nennen, zum anderen der gescheiterte Staatsstreich im Homeland Bophuthatswana (April 1994), der ebenfalls aus Reihen des AWB lanciert wurde. ${ }^{28} \mathrm{Zu}$ betonen ist die ideologische, personelle und organisatorische Nähe des AWB zu den rechten Afrikaner-Parteien CP und AVU und deren Dachorganisation AVF. ${ }^{29}$ Der gescheiterte Coup entfernte ironischerweise die letzten Hindernisse auf dem Weg zur ersten demokratischen Wahl: Er führte zur Spaltung der weißen Rechten in einen legalistischen Flügel, die Freedom Front, und die verbliebenen Militanten der AWB. Bis dahin gefährdete die CP durch überraschende Erfolge in Nachwahlen zunehmend die Position der NP, deren Mehrheit im Parlament zusehends kleiner wurde.

Für ein antiextremistisches Parteiverbot hätte in der Phase der Verhandlungen über eine neue politische Ordnung (1991-1994) durchaus Anlass bestanden. Die COSAG Parteien besetzten zwar keine effektiven Veto-Positionen im Prozess, hatten jedoch ein erhebliches „Spoiling-Potential“ ${ }^{30}$ Neben den bereits genannten Akteuren ist mindestens noch der Pan Africanist Congress zu nennen. ${ }^{31}$ Dieser stand bis 1993 ebenfalls außerhalb des prä-konstitutionellen Bogens, da er eine Reform des Systems durch Verhandlungen ablehnte: „Slaves have nothing to gain from negotiating with their masters (...) We do not need reform. We need a complete overhaul of the entire economic and political system". ${ }^{32}$

Auch wenn CP, PAC, AVU und phasenweise IFP als extremistische Parteien eingeordnet werden können, von denen signifikante Risiken für den Transformationsprozess ausgegangen sind, so war ihr Verbot aber nicht praktikabel und legitimierbar im Licht der Zielsetzung des Verhandlungsprozesses. Die Gründe, von einem Verbot dieser Gruppen im Transformationsprozess abzusehen, werden in Kapitel 4 betrachtet. An dieser Stelle kann zusammenfassend nur so viel festgestellt werden, dass objektive Grundlagen sowohl eines

Klerk später auch zugeben musste. Vgl. Richard A. Wilson, Justice and Legitimacy in the South African Transition, in: Alexandra Barahona de Brito / Carmen Gonzales-Enriquez/ Paloma Aguilar (eds.), The Politics of Memory. Transitional Justice in Democratizing Societies, 2001, S. 196.

Spitz / Chaskalson, oben Fn. 20, 244 ff.

CP: Conservative Party; AVU: Afrikaner Volksunie, AVF: Afrikaner Volksfront; AWB: Afrikaner Weerstandsbeweging.

Ein „Spoiler“ oder „Störenfried“ zeichnet sich wesentlich durch sein negatives Blockade- oder Sabotagepotential aus. Dadurch wird er in die Lage versetzt Verhandlungslösungen zu verhindern, ohne jedoch selbst Perspektiven jenseits des Status Quo aufzeigen zu können. Im Negativfall führt das Handeln von Spoilern sogar zu einem Rückfall hinter den Status Quo. Vgl. dazu Stephen John Stedman, Spoiler Problems in Peace Processes, International Security 22 (1997), S. 5-53 und Ulrich Schneckener, Warum manche den Frieden nicht wollen. Eine Soziologie der „Störenfriede", SWP Forschungsgruppe Globale Fragen-Diskussionspapier 1/2003.

31 Prof. Robert Mattes verweist auf die destruktive Haltung des PAC in weiten Phasen des. Verhandlungsprozesses (Interview, Cape Town, 03.06.2008).

32

Zitiert nach Ebrahim, oben Fn. 23, S. 43. 
anti-extremistischen als auch eines negativ republikanischen Parteiverbots durchaus bestanden.

Auf der „weißen“ Rechten gab es Bestrebungen, den Demokratisierungsprozess zu verhindern und das Apartheidsystem zumindest territorial begrenzt aufrecht zu erhalten. Die „schwarze“ ANC-Opposition zeigte ebenso mannigfache Bestrebungen, den Transformationsprozess zu unterlaufen. Ihr konservativ-ethnisch orientierter Teil wollte eine zu starke Machtballung im Zentrum verhindern und seine unter dem Apartheidregime bezogenen Machtpositionen nicht verlieren. IFP beispielsweise stellte mit der Forderung nach einer Sonderrolle KwaZulu Natals möglicherweise die territoriale Integrität des Landes in Frage. Ihre Forderung nach Gleichstellung des „Traditional law“ in der Rechtshierarchie forderte zudem den unbedingten Geltungsanspruch des Demokratie- und Rechtsstaatsprinzips heraus. Die afrikanistische Linke stand einer Zusammenarbeit mit der alten Regierung überhaupt kritisch oder gar feindlich gegenüber und griff den ANC aufgrund dessen Konsensorientierung an. Ein negativ republikanisches Parteiverbot hätte sich auf die konkrete Negativfolie der Apartheid beziehen und diese systematisch ausschließen können. Da negativer Republikanismus eine bestimmte Form der Vergangenheitspolitik darstellt, ist erstens zu prüfen, inwiefern Südafrika auf alternative Formen zurückgegriffen hat und zweitens, warum bestimmte Varianten der Vergangenheitspolitik gewählt und andere verworfen wurden. Da die Institutionenwahl in verhandlungsgesteuerten Transitionsprozessen durch die Interaktion der ,alten“ und ,neuen“ Eliten maßgeblich geprägt wird, verdient dieses Verhältnis in der Analyse besondere Beachtung.

\section{Die Demokratiekonzeption der Südafrikanischen Verfassung}

Die südafrikanische Verfassung verbindet zwei grundlegende Prinzipien. Das erste Prinzip kommt in den „Founding Provisions“ der Verfassung zum Ausdruck, als „Supremacy of the Constitution and the Rule of Law “ und in der „Bill of Rights“, jeweils gewährleistet durch das unabhängige Verfassungsgericht. Die Unveränderlichkeit dieses Rechtsstaatsprinzips und die kategoriale Bindung allen positiven Rechts daran zeichnet die Verfassung als ,substantive democracy“ aus. Das zweite Prinzip besteht in dem durchweg liberalen Verständnis der Rechte, welches bürgerlichen und politischen Rechten unbedingte Geltung zuerkennt. Art. 18 der Verfassung bekennt schlicht: „Every person has the right to freedom of association“. Art. 19 ("Political Rights”) gewährleistet das Recht:

(a) to form a political party; (b) to participate in the activities of, or recruit members for, a political party; (c) to campaign for a political party or cause".

Darüber hinaus gewährleistet Art. 19 das uneingeschränkte aktive und passive Wahlrecht. Diskriminierung hinsichtlich rassischer oder ethnischer Abstammung sowie der Geschlechtszugehörigkeit verbietet Art. 9 (,Equality“). Auf diskriminierende Praktiken haben sowohl der Staat als auch Privatpersonen zu verzichten.

Die Einschränkung von Rechten („Limitation of Rights“) wird in Art. 36 an enge Kriterien gebunden. Dennoch erscheinen die Formulierungen der „limitation-clause“ auf den 
ersten Blick Ansätze für mögliche Einschränkungen politischer Assoziationsfreiheit zu bieten. Die Interim Constitution erlaubte in Art. 33 (1) die Einschränkung von Rechten unter der Bedingung, dass diese Einschränkung ,reasonable“ und ,justifiable“ „,in an open and democratic society" sei. Für die Einschränkung einiger Rechte, unter anderem die in unserem Fall relevanten „Freedom of Association“ und die „Political Rights“ gilt außerdem das Kriterium „necessary“, welches besonderes hohe Hürden gegenüber einer Einschränkung nahelegt. Hierzu merkt Stuart Woolman im Verfassungskommentar an: „Rights which receive, reasonable and necessary' protection should receive the greatest judicial solicitude". 33 In der Limitation Clause der Final Constitution (Art. 36) wurde das Kriterium der Notwendigkeit gestrichen, was Woolman zufolge zu einer Enthierarchisierung verfassungsmäßiger Rechte führte. Die Tendenz der Interim Constitution ,to privilege liberal and procedural rights over more egalitarian guarantees“" ${ }^{34}$ sei dadurch entfallen. Gemäß dem Verständnis der analog formulierten Rechte im ,Internationalen Pakt über bürgerliche und politische Rechte“ könnte dies auf eine Erleichterung der Einschränkung verfassungsmäßiger Rechte hinweisen. ${ }^{35}$ Diese Interpretation lehnen jedoch sowohl Woolman als auch Currie und de Waal ab. ${ }^{36}$ Die Neufassung wird hingegen als terminologische Straffung im Sinne eines ,non-preferential treatment of all rights under the limitation clause “6 37 bewertet.

Inwiefern eröffnet die Interpretation der einschlägigen Art. zur Assoziationsfreiheit und zu den politischen Rechten die Möglichkeit von Parteiverboten? Stuart Woolman grenzt im Kommentar zu Art. 18 die Konzeption der Assoziationsfreiheit scharf von der südafrikanischen Tradition der Assoziationsverbote ab. Die repressive Vorgeschichte wird in Anspruch dafür genommen, dass ,great caution should be exercised before resort is had to the banning of any political entity". 38

Assoziationsverbote werden streng an das Handeln von Organisationen gebunden. Bestimmte Willensbekundungen, die demokratische Ordnung stürzen zu wollen, reichen nicht als Rechtfertigung aus. Mögliche Verbote beziehen sich somit nicht auf militante Demokratiegegner, sondern auf solche Akteure, die über reale Machtmittel zur Herbeiführung eines Umsturzes verfügen, und diese Intention durch Gewaltanwendung auch belegen: „This rule draws a distinction between associations which merely advocate the government's overthrow - which deserve at least prima facie protection - and associations which demonstrate through military preparation and action that they are bent on non-peaceable

Stuart Woolman, Section 12: Limitation, in: Matthew Chaskalson (ed.), Constitutional Law of South Africa, Cape Town 1999, S. 37.

Woolman, oben Fn. 33, S. 13. Siehe Woolman, oben Fn. 33 und Iain Currie / Johan de Wall, The Bill of Rights Handbook, Cape Town 2005 (5th edition). 
governmental change“. ${ }^{39}$ Es müssen also konkrete Gewalthandlungen massiven Ausmaßes vorliegen, um Organisationen zu verbieten, die militante Ablehnung der demokratischen Ordnung durch diese reicht hingegen nicht aus. Damit fallen Parteien als Ziele von Assoziationsverboten weitgehend weg, zumindest falls sie nicht als Träger paramilitärischer Gewalt in Erscheinung treten. 40

Allerdings muss das Recht der Assoziationsfreiheit im Kontext der möglichen Einschränkungen der „Freedom of Expression“ interpretiert werden. Die Meinungsfreiheit erfährt in Art. 16 (2) eine Einschränkung in drei Fällen:

(a) Propaganda for war; (b) Incitement of imminent violence; (c) Advocacy of hatred that is based on race, ethnicity, gender or religion, and that constitutes incitement to cause harm.

Können aus diesen Einschränkungen der Äußerungsfreiheit auch Einschränkungen der Organisationsfreiheit abgeleitet werden? Der Verzicht, in Art. 18 („Freedom of Association“) eine zur Äußerungsfreiheit analoge Formulierung zu wählen und Einschränkungsgründe zu enumerieren, spricht dagegen, der Normen zur Äußerungsfreiheit auf die Assoziationsfreiheit zu übertragen. Können zudem Parteien überhaupt für Äußerungen ihrer Mitglieder (,vicarious liability“) verantwortlich gemacht werden? Die südafrikanische Verfassungsrechtslehre interpretiert mehrheitlich die Grundrechte eher individualisierend, was eine Übertragbarkeit auf Organisationen nicht nahe legt. ${ }^{41}$

„Political Rights“ (Art. 19) bilden ,,in the light of the historical context (...) a central pillar of a representative democracy“. ${ }^{42}$ Auch hier soll die geschichtliche Erfahrung ein besonders tolerantes Rechtsverständnis begründen. Neben dieser partikularen Erfahrung wird auch auf die universelle Dimension eines ,emerging right to democratic governance“ verwiesen. Das Recht, politische Parteien zu gründen, in ihnen mitzuarbeiten und für sie zu werben, hebt Art. 19 hervor. Parteien werden somit gegenüber der „normalen“ Assoziationsfreiheit besonders ausgezeichnet, ohne Anlegung besonders strenger Maßstäbe an ihre innere und äußere Gestalt. Die Interpretation von Art. 19 im Kontext der Art. 9 („Equality“) und 16 („Freedom of Expression“) ermöglicht jedoch bestimmte Zugangs-Regularien zur Teilnahme an Wahlen als der Kerninstitution der repräsentativen Demokratie und darüber auch zur öffentlichen Parteienfinanzierung, die ihrerseits Verfassungsrang genießt (Art. 52).

Das Recht zur Parteibildung unterliegt keinen Einschränkungen, auch nicht durch die einschlägige Gesetzgebung des Electoral Act (EA) und des Electoral Commission Act (ECA). Das gilt nicht für das ,right to campaign“. Art. 99 des Electoral Act normiert die Zustimmung jeder sich an einer Wahl beteiligenden Partei zum Electoral Code. Der Electo-

40

41

Woolman, oben Fn. 38, S. 7.

Currie / de Waal, oben Fn. 36, 427.

Johan de Waal, Section 23: Political Rights, in: Matthew Chaskalson (ed.), Constitutional Law of South Africa, Cape Town 1999, S. 6.

De Waal, oben Fn. 41, S. 2. 
ral Code ist dem Electoral Act als Ergänzung beigefügt und beschränkt die Äußerungsfreiheit von Parteien analog zu Art. 16. Bei Verstößen gegen diesen „Code of Conduct“ sieht Art. 96 EA Sanktionen vor, die bis zur Nichtregistrierung von Parteien reichen können (Art. 96 (2) (i)). ${ }^{43}$ Die „Independent Electoral Commission (IEC)“ dokumentiert zwölf Fälle von verweigerter Registrierung auf nationaler und Provinz-Ebene bis Juni $2008{ }^{44}$ Bislang sind keine Fälle bekannt, in denen Parteien aufgrund von Wahlkampfäußerungen wegen ethnischer Diskriminierung, ausgeschlossen worden wären. Verweigert wurden Registrierungen bislang bei Nichterfüllung der in Art. 15 ECA normierten formalen Anforderungen oder Verstößen gegen prozedurale Bestimmungen des Art.s 16 (1) a (i) ECA (s.u.). ${ }^{45}$

Der Electoral Commission Act ermöglicht dem Chief Electoral Officer (Geschäftsführer der IEC) prinzipiell, Parteien nicht zu registrieren, die bestimmten Anforderungen nicht genügen. Auch hier wird auf die Terminologie von Art. 9 und Art. 16 der Verfassung zurückgegriffen:

Sec 16. Electoral Commission Act

(1) The chief electoral officer may not register a party in terms of section 15 , if:

(a) a proposed name, abbreviated name, distinguishing mark or symbol mentioned in the application:

(i) resembles the name, abbreviated name, distinguishing mark or symbol, as the case may be, of any other registered party to such extent, that it may deceive or confuse voters ; or

(ii) contains anything which portrays the propagation or incitement of violence or hatred or which may cause serious offence to any section of the population on the grounds of race, gender, sex, ethnic origin, colour, sexual orientation, age, disability, religion, conscience, belief, culture or language.

Zusammengefasst kennt die Verfassung der Republik Südafrika kein Parteiverbot, das auf eine Auflösung von Parteien hinauslaufen würde. Begründet durch die Interpretation von Art. 19 (1), kann Parteien allerdings ihre Registrierung zu Wahlen verweigert werden. Das hat insbesondere den Wegfall öffentlicher Parteienfinanzierung zur Folge. ${ }^{46}$ Statt von einem Verbot lässt sich daher nur von einer Suspendierung sprechen.

\section{E. Warum gibt es kein Parteiverbot in der südafrikanischen Verfassung?}

Die Südafrikanische Verfassung bekennt sich zu einer liberal-toleranten Demokratiekonzeption, trotz der in Kapitel 2 diskutierten historischen Gründe, die durchaus in der verfas-

Eine schwere Sanktion, da die mandatsgebundene öffentliche Parteienfinanzierung wegfiele.

Vgl. die Homepage der Independent Electoral Commission (http://www.elections.org.za/ Registered_Parties/Selection_Party.asp).

Diese Informationen beruhen auf Angaben der Independent Electoral Commission sowie Experteninterviews mit Hugh Corder, Robert Mattes und Richard Calland an der University of Cape Town. Betroffen von der Nichtregistrierung war beispielsweise eine Labour-Party, deren Registrierung aufgrund der Existenz einer anderen Labour Party nicht angenommen wurde.

De Waal, oben Fn. 41, S. 7. 
sungsgebenden Situation für die Einführung von Parteiverboten hätten geltend gemacht werden können. Im folgenden sind zunächst, basierend auf einer im April und Mai 2007 durchgeführten Expertenbefragung ${ }^{47}$, drei Hypothesen zu entwickeln, die den Verzicht auf das Instrument Parteiverbot erklären können. Unter Einbezug der Expertenbefragung, von Sekundärliteratur und im Mai/Juni 2008 generierten Interviewdaten ${ }^{48}$ sind die Hypothesen dann zu überprüfen.

\section{Hypothesen}

Die Transformation folgte in Südafrika trotz aller Hindernisse einem konsensorientierten oder koordinierten Modell, das der Einbeziehung möglichst vieler und heterogener Akteure eine wichtige Rolle zuwies. Analysen dieses Modells verweisen gleichermaßen auf die Bedeutung von Akteursüberzeugungen ${ }^{49}$, auf das rationale Kalkül der Beteiligten ${ }^{50}$ und auf die Mechanismen der Transformation, die einen bestimmten ,constitutional choice“ als Ergebnis nahe legen. Die wichtigsten theoretischen und empirischen Alternativen zum Konsensmodell stellen die Reform von oben und der Zusammenbruch des alten Regimes dar, der sowohl durch eine Revolution als auch durch äußere Intervention bewirkt werden kann. ${ }^{51}$ Die politische Konstellation in Südafrika ermöglichte allerdings eine koordinierte Transition.

Die Einführung von Parteiverboten, respektive der Verzicht darauf, kann nur im allgemeinen Kontext der Verfassungsgebung erklärt werden, da sich im geschriebenen Verfassungstext gleichermaßen historische Erfahrungen, die Überzeugungen der handelnden Akteure und der Einfluss konkurrierender sozialer Kräfte widerspiegeln. Dieses Verfas-

Im März/April 2007 wurde eine Befragung südafrikanischer Experten durchgeführt, an der sich folgende Personen beteiligten: Prof. Heribert Adam (Simon Fraser University, Ontario/Kanada), Prof. Kogila Adam-Moodley (University of British Columbia, Kanada), Prof. David Dyzenhaus (Faculty of Law, University of Toronto), Prof. Pierre du Toit (Department of Political Science, University of Stellenbosch), Prof. Hermann Giliomee (University of Stellenbosch), Prof. Courtney Jung (New School of Social Research, New York), Prof. Heinz Klug (Law School, University of Wisconsin), Prof. Robert Mattes (Department of Political Studies, University of Cape Town).

Während eines Forschungsaufenthaltes in Südafrika im Mai/Juni 2008 konnten Experteninterviews mit den folgenden Personen geführt werden: Dr. Werner Böhler (Repräsentant der Konrad Adenauer Stiftung, Johannesburg), Hendrik Bussiek (Journalist/Politikberater, Kapstadt), Prof. Hugh Corder (Faculty of Law, University of Cape Town), Prof. Richard Calland (Faculty of Law, University of Cape Town), Prof. Robert Mattes (Department of Political Studies, University of Cape Town), Ursula Scheidegger (Department of Political Studies, University of the Witwatersrand, Johannesburg/EISA, Johannesburg).

50 Sparks, oben Fn. 22.

Sisk, oben Fn. 18

51 Für eine überzeugende theoretische Herleitung und empirische Anwendung dieser Kategorien vgl. János Kis, Between Reform and Revolution, Eastern European Politics and Societies 12 (1998), S. 300-383 und daran anschließend Andrew Arato, The Occupation of Iraq and the difficult Transition from Dictatorship, Constellations 10 (2003), S. 408-424.. 
sungsverständnis, in welchem das Staatsrecht als Ausdruck sozialer Konstellationen zu verstehen ist ${ }^{52}$, legt eine Untersuchung nahe, die eine bestimmte institutionelle Wahlhandlung (Verzicht auf eine Parteiverbotsnorm) nicht isoliert, sondern in ihrem historischen Kontext betrachtet.

Experten des südafrikanischen Transformationsprozess erhielten einen offenen Fragebogen zum Thema „Parteiverbot“. Gefragt wurde zum ersten, ob im Rahmen des Demokratisierungsprozesses über die Einführung von Parteiverboten in eine neue Verfassung diskutiert wurde, von wem solche Vorschläge gemacht wurden, auf welchen Typ von Parteien sie sich bezogen und welche Gründe geltend gemacht wurden. Fehlte eine solche Parteiverbotsdebatte, wurde zweitens nach möglichen Gründen für deren Ausbleiben gefragt. Die dritte Frage bezog sich darauf, ob das Thema seit Inkraftsetzung der endgültigen Verfassung in der verfassungspolitischen Debatte aufgeworfen wurde. Basierend auf den Antworten konnten drei Hypothesen formuliert werden:

1. Vergangenheitspolitisch wurde auf Parteiverbote verzichtet, weil Partei- und Organisationsverbote wichtige Repressionsinstrumente im Apartheidsystem darstellten. Der einschränkungsfreien Partizipation in politischen Parteien und deren Rolle im politischen System wurde daher ein hohes Maß an Legitimität zugebilligt.

2. Der südafrikanische Weg zur Demokratie folgte dem Modell der „,coordinated transition“. Inklusivität und breiter Konsens sind wichtige strukturelle Bestandteile dieses Modells. Parteiverbote wären hier ein systemfremdes Element.

3. Der politische Extremismus bedrohte trotz Präsenz entsprechender Akteure (vgl. Kapitel 2) die neu entstehende Demokratie nicht ernstlich. Der Nutzen eines Parteiverbots stünde außer Verhältnis zu seinen Kosten für die liberale Verfassungskonzeption.

II. Analyse

Die Analyse für die Gründe des Verzichts auf eine Parteiverbotsnorm wird erstens darauf eingehen, warum ein negativ-republikanisches Parteiverbot in vergangenheitspolitischer Perspektive keine Option darstellte. Hier spielen insbesondere die Überzeugungen der handelnden Akteure eine bedeutsame Rolle. Zweitens sollen zentrale Strukturen des Transformationsprozesses untersucht werden. Schließlich wird geprüft, warum trotz der offensichtlichen Bedrohung des Demokratisierungsprozesses durch eine Vielzahl von Organisationen auch die anti-extremistische Variante eines Parteiverbots nicht zum Zuge kam. Diese Analyse betont die Akteurkonstellation im Verfassungsgebungsprozess sowie die Akteurinteressen und wird abschließend flankiert durch eine machtstrategische Zusatzüberlegung, die auf die im Transformationsprozess bereits absehbare Struktur des südafrikanischen Parteiensystems nach 1994 abhebt. 1972. 
Analytisch kann die Erklärung für den Verzicht auf Instrumente der „militant democracy“ auf drei Ebenen verortet werden. Auf der strukturellen Ebene können die „Eigengesetzlichkeiten“ des südafrikanischen Transformationstyps herangezogen werden. Auf der Ebene der Akteurskonstellation und Akteursinteressen steht das rationale Kalkül der Akteure im Transformationsprozess im Mittelpunkt. Die Akteursüberzeugungen bilden schließlich tieferliegende Wert- und Weltvorstellungen auf Seiten der handelnden Eliten ab. Während die Hypothesen 1 und 2 weitgehend bestätigt werden können, bedarf Hypothese 3 zumindest einer Modifikation. Während die entstehende Demokratie im Zeitraum zwischen der Aufnahme offizieller Gespräche zwischen der NP-Regierung und dem ANC (1990) und der Wahl der Verfassungsgebenden Versammlung (1994) unter erheblichem Druck ,extremistischer“ Akteure stand, schwächte sich dieser nach 1994 ab durch Einbindung oder Marginalisierung.

\section{II.1 Vergangenheitspolitik und Akteursüberzeugungen}

In vergangenheitspolitischer Hinsicht betonen mehrere Interviewpartner, dass der Verzicht auf Assoziationsverbote in der Verfassung eine bewusste Abkehr von der Vergangenheit darstellte. Robert Mattes erwähnt zum Konzept der „liberatory intolerance“: „This idea never got very far at all, mostly because it smacked too much of what had gone on before“. ${ }^{53}$ Mit der Weichenstellung für einen ausgehandelten Regimewechsel entfielen die notwendigen Voraussetzungen einer Leitbildfunktion der ,liberatory intolerance“, denn das Konzept basierte auf der Annahme eines von unten erzwungenen Regimewechsels oder eines Regimezusammenbruchs. In der südafrikanischen Transformation erfolgte hingegen eine „Läuterung“ des wichtigsten Trägers der alten Ordnung, der NP, durch deren maßgebliche Mitwirkung an der Schaffung einer neuen Ordnung. Intoleranz erfuhr zudem eine gänzliche Umkodierung. Intoleranz galt bereits im Transformationsprozess als ein zu überwindendes Übel und mögliches Hindernis für eine erfolgreiche Demokratisierung. ${ }^{54}$ Gibson führt zum Konzept der "liberatory intolerance” aus: „Theories such as this divided supporters of apartheid and those struggling against it into categories of enemies and friends, evil and good, and lent a certain justification (if not imperative) to intolerance". ${ }^{55}$ Herman Giliomee sieht die negative Erfahrung mit Parteiverboten als den wichtigsten Grund dafür, dass deren neuerliche Einführung nicht erwogen wurde. "Inclusion of all political actors became the overriding goal of constitutionmaking“, in Abgrenzung zur Apartheid als „exclusionary system“. 56 Da die Anti-Apartheid Opposition in großer Mehrheit sich für eine „culture of inclusion“ ausgesprochen hatte, hätte eine ,policy of prohibition“ die

53

54

55

56

Interview mit Robert Mattes (Cape Town, 03.06.2008).

Siehe Institute for a Democratic Alternative for South African, Preparing for Democracy, Die Suid-Afrikaan 43 (1993).

Gibson, oben Fn. 16, S. 26.

Fragebogen von Herman Giliomee. 
Glaubwürdigkeit der zentralen Akteure in Frage gestellt. ${ }^{57}$ Tatsächlich hatte das ApartheidRegime extensiv auf das Instrument des Organisationsverbots zurückgegriffen. Als erste Anti-Apartheid-Partei nach der Machtübernahme der „Afrikaner-Nationalisten“ wurde 1950 die SACP (South African Communist Party) durch den „Suppression of Communism Act" verboten. ${ }^{58} 1960$ folgten Verbote des ANC und des PAC, 1977 des BCM (Black Consciousness Movement) und 1988, noch in der Spätphase der Apartheid, der UDF (United Democratic Front). Die UDF war eine Dachorganisation von Apartheidgegnern unterschiedlicher Herkunft und warb explizit für ein multiethnisches Südafrika. Die hohe symbolische Bedeutung der Organisationsverbote wird daran deutlich, dass sie als zentrale Herrschaftsinstrumente des alten Systems interpretiert werden. So bezeichnet Nelson Mandela in seiner Autobiographie ${ }^{59}$ den „Suppression of Communism Act“" als einen der drei Grundpfeiler der Apartheid, neben dem „Population Registration Act“ und dem „Group Areas Act“ (Mandela 2006, 160). ${ }^{60}$ Daran wird die immense symbolische Aufladung des Verbots-Instrumentes deutlich. Daher hätte die Verhängung von Organisationsverboten wie eine Fortschreibung des alten Systems gewirkt und der Reputation und Legitimation der Transformationsakteure schweren Schaden zugefügt.

Die NP-Regierung hatte 1990 die noch bestehenden Organisationsverbote (ANC, PAC, SACP) aufgehoben. Neuerliche Verbote wären vor diesem Hintergrund schwer zu rechtfertigen gewesen und hätten sich höchstwahrscheinlich noch konfliktfördernd ausgewirkt. Sowohl auf Seiten der „,weißen“ (rechten, pro Apartheid) als auch der „,schwarzen“ (entweder ethnisch-konservativen oder links-afrikanistischen) Opposition im Verhandlungsprozess wären Gewalt und Sezession in noch stärkerem Maße propagiert worden, als dies sowieso schon der Fall war. Hier wird das Legitimitätsargument durch ein Nützlichkeitsargument ergänzt. Parteiverbote hätten dem gemeinsamen Ziel der dominanten Akteure, der friedlichen Demokratisierung des Apartheidsystems im Rahmen der bestehenden legalen Ordnung, geschadet. So stand die NP beispielsweise unter dem Druck der CP, die in den Wahlen der achtziger Jahre zunehmend ihren Stimmenanteil ausbauen konnte und nach 1990 alle Nachwahlen gewann. Repressive Maßnahmen gegenüber dieser entschiedenen Pro-Apartheid-Partei hätten die Wahrscheinlichkeit des Scheitern des Verhandlungsprozesses signifikant erhöht. Gleiches gilt für die Verhandlungsgegner auf der anderen Seite. Deren etwaige Ausschaltung durch die NP-Regierung hätte auf Seiten des ANC zu erheblichen Legitimationsproblemen geführt, da dieser unweigerlich in den Verdacht des „Verrats“ gekommen wäre. Zu beachten ist auch die organisatorische Einbindung eines großen

Ebrahim, oben Fn. 23, S. 13.

59 Nelson Mandela, Der lange Weg zur Freiheit, Hamburg 2006, S. 160.

60

Der „Population Registration Act” legte die Rassenzugehörigkeit jedes Südafrikaners fest, der „Group Areas Act“ wies den jeweiligen Gruppen feste Wohngebiete zu, wodurch die Segregation der ethnischen Gruppen zementiert wurde. 
Teils der radikalen Linken in den ANC unter dem Dach der tripartistischen Allianz. ${ }^{61}$ Die SACP fiel somit als möglicher „Spoiler“ weg.

Ein wichtiges Element stellen sicherlich auch gewisse Übereinstimmungen der Transformationseliten hinsichtlich theoretischer und konstitutioneller Überzeugungen dar. Die prinzipielle Orientierung am britischen Konstitutionalismus bedingte die Legitimität eines „political party model of democracy“ und des normativen Ideals des „limited government under the rule of law“. ${ }^{62}$ Diese Beobachtung gilt insbesondere für die Akteure in den bedeutsamen Technical Committees im Multi Party Negotiation Process. Nach Aussage eines Mitglieds des „Technical Committee on the Bill of Rights“ seien Parteiverbote dort niemals debattiert worden. ${ }^{63}$ Ihre Basis finden diese Überzeugungen in einer im afrikanischen Vergleich einzigartigen parteipolitischen Tradition, in der die ersten Parteien bereits um 1900 gegründet und erste Wahlen bereits 1910 abgehalten wurden. Diese Tradition liefert auch eine Erklärung für die liberale Form der Parteienregulierung in funktionaler Hinsicht. $^{64}$

Schließlich ist in vergangenheitspolitischer Perspektive noch die Frage zu stellen, inwiefern funktionale Äquivalente $\mathrm{zu}$ einem negativ-republikanischen Parteiverbot zu finden sind. Ruti Teitel unterscheidet verschiedene Typen von „transitional justice“, wobei sie „,militant democray“ als eine Form von ,administrative justice“ verortet. ${ }^{65}$ Die südafrikanische Wahrheits- und Versöhnungskommission ${ }^{66}$, deren Resultate sehr kontrovers beurteilt werden, stellt ein Instrument zur Generierung historischer Gerechtigkeit (,historical justice“) dar. An dieser Stelle kann nicht die Diskussion über die TRC rekonstruiert werden, allerdings scheint es interessant die Frage aufzuwerfen, ob Wahrheitskommissionen eine Form des Transformationsmanagements darstellen, die ,robustere“ Instrumente wie Parteiverbote ausschließen bzw. ersetzen. Hugh Corder beschreibt „Lustration“ und Organisationsverbote auf der einen und „Wahrheits- und Versöhnungskommissionen“ auf der anderen Seite als distinkte Modelle zur Herstellung von ,transitional justice“, die in der südafrikanischen Debatte durchaus als Alternativen wahrgenommen wurden, wobei sich schließlich das Kommissionsmodell durchgesetzt habe. ${ }^{67}$

Diese besteht aus der ANC Kernorganisation, der SACP und dem Gewerkschaftsverband COSATU.

Ebd.

Für die konkrete Verfassungsgebung waren indes andere Modelle als das britische relevanter. Insbesondere das Deutsche Grundgesetz und die Kanadische Verfassung, aufgrund der ausdifferenzierten Grundrechtskapitel, der institutionellen Gewaltenteilung und der zentralen Rolle der Verfassungsgerichte, hatten eine Vorbildfunktion für die Südafrikanischen Verfassung. Teitel, oben Fn. 14. 


\section{II.2 Strukturen der Transformation}

Aus der Perspektive der Transformationsforschung gerät an dieser Stelle der Charakter des südafrikanischen Systemwechsels in den Blick. Dieser entspricht einer ausgehandelten oder koordinierten Transformation ${ }^{68}$ mit folgenden Merkmalen: Die Ursachen des Wandels liegen in der inneren Krise des Apartheidsystems begründet, das zunehmend auch innerhalb der bevorzugten Bevölkerungsgruppe an Legitimität verlor. Das Ende des Kalten Krieges und die Sanktionen gegen das Regime können darüber hinaus als ein Wegfall externer Unterstuitzung gewertet werden. ${ }^{69}$ Die ,attidudes of the outgoing elite toward democracy“ waren überwiegend positiv oder zumindest von einer mehr oder weniger gleichgültigen Akzeptanz geprägt. ${ }^{70}$ Ein weiterer Faktor war die Existenz legitimer Verhandlungspartner seitens der „Herausforderer“, namentlich des ANC und seiner Verbündeten in der tripartistischen Allianz. Diese Verhandlungspartner hatten zudem bereits lange vor Beginn der Transformation begonnen Kontakte zu knüpfen, die zwischen 1985 und 1989 sukzessive ausgeweitet wurden. ${ }^{71}$ Dabei entwickelte sich ein Vertrauensverhältnis, das in der ,heißen Phase“ des Systemwechsels auch die Überwindung von Krisen ermöglichte. Die Übereinstimmung in Grundfragen zwischen der ANC-Führung und dem Afrikaner-Establishment kommt in diversen Dokumenten zum Ausdruck, angefangen vom „Groote Schuur Accord“ (4. Mai 1990), über die „Declaration Of Intent“ der ersten „Convention for a Democratic South Africa“ CODESA I (21. Dezember 1991) bis zum „Agreement To Resume Bilateral Negotiations“ (25. November 1992) zwischen der Regierung und dem ANC. ${ }^{72}$

Nach Andrew Arato stellt die koordinierte Transition den vorzugswürdigen Demokratisierungspfad dar. ${ }^{73}$ Sie führt idealtypisch zum Wechsel der sozialen Legitimitätsbasis einer politischen Ordnung bei gleichzeitiger Kontinuität der formalen Legalität des überwundenen Systems. Im südafrikanischen Fall blieb die „Legalität“ dadurch erhalten, dass nur die weiße Bevölkerung in einem Referendum im März 1992 die Regierung ermächtigte, Verhandlungen mit dem Ziel der Schaffung einer demokratischen Verfassung für Südafrika zu führen. Die Interimsverfassung, der die endgültige Verfassung im Wesentlichen folgt, wurde 1993 von der Mehrheitsfraktion des, ,weißen“ Parlamentes ratifiziert. Auf ihrer Basis

Wolfgang Merkel / Peter Thiery, Systemwechsel, in: Hans-Joachim Lauth (Hrsg.), Vergleichende Regierungslehre, Wiesbaden 2006, S. 168.

Vgl. John Hoffman, Legitimate Intervention and Illegitimate States. Sanctions against South Africa, in: Ian Forbes / Mark Hoffman (eds.): Political Theory, International Relations, and the Ethics of Intervention, S. 157-166.

Jorge Heine, Institutional Engineering in New Democracies, in: Ursula J. van Beek (ed.) Democracy under Construction. Patterns from four Continents, Bloomfield Hills / Opladen 2005, S. 71.

Siehe Sparks, oben Fn. 22, S. 39 ff. und Ebrahim, oben Fn. 23, S. 19 ff..

72

Ein Abdruck der wichtigsten Dokumente findet sich bei Ebrahim, oben Fn. 23.

73

Arato, oben Fn. 51, S. 412. 
wurde die „Constitutional Assembly“ gewählt, die im Mai 1996 die von ihr ausgearbeitete endgültige Verfassung annahm. ${ }^{74}$

Ein solcher Transitionstyp ist allerdings an vergleichsweise anspruchsvolle Voraussetzungen gebunden, vor allem an ,social forces capable of negotiation“. Da in Südafrika im wesentlichen die beiden ältesten afrikanischen Parteien ${ }^{75}$ die Transformation aushandelten, kommt Heine zu dem Urteil: ,South Africa would potentially be in the best position to consolidate its democratic institutions as its transition was very much a negotiated one (in which the rules of the previous regime were not broken) and the attitude of the outgoing elite was in favor of democracy." 76 Die neue Regierung konnte nach 1994 also auch auf eine funktionierende und weitgehend loyale Administration zurückgreifen. Lustration wurde als vergangenheitspolitisches Modell nicht oder nur in sehr eingeschränktem Rahmen angewendet. Die Justiz blieb weitgehend in Amt und Würden und auch in den ,,senior civil service ranks" gab es nur vereinzelt vorgezogene Pensionierungen oder den ,golden handshake". Ein unmittelbar erzwungener Elitenaustausch erfolgte nicht. ${ }^{77} 78$

Der inklusive Charakter der Verhandlungen baute sehr stark auf den Konsens aller Beteiligten, auch derjenigen, die zeitweilig aus dem Prozess ausstiegen, d.h. die COSAG Parties. ${ }^{79}$ Damit folgten die Akteure dem Leitbild der Offenheit und Inklusivität, das etwa in der „Declaration Of Intent“ zu CODESA I formuliert wird: „We agree that the present and future participants shall be entitled to put forward freely to the Convention any pro-

Diese konnte wiederum erst nach einer Revision des Verfassungstextes durch das Verfassungsgericht in Kraft treten.

Zum Stammbaum der NP vgl. Hennie Kotzé, A Consummation devoutly to be wished? The Democratic Alliance and its potential Constituencies, in: Roger Southall (ed.), Opposition and Democracy in South Africa, London 2001, S. 118 f.; zum ANC vgl. Tom Lodge, The ANC and the Development of Party Politics in modern South Africa, Journal of Modern African Studies 42 (2004), S. 189-219.

Vgl. oben Fn. 70.

Interview mit Hugh Corder (Cape Town, 04.06.2008).

Allerdings soll an dieser Stelle dem möglichen Missverständnis vorgebeugt werden, in Südafrika habe kein struktureller und personeller Wandel des öffentlichen Sektors stattgefunden. Der ,racial redress“ soll jedoch in erster Linie durch ,affirmative action“ erreicht werden. Diese findet ihre rechtliche Grundlage in der Selbstverpflichtung der Interimsverfassung, ,the public service shall be broadley representative of the South African community" und im Employment Equity Act von 1998, so Mcebisi Ndletyana, Affirmative Action and the Public Service, in: Adam Habib / Kristina Bentley (eds.), Racial Redress and Citizenship in South Africa, Cape Town 2008, S. 77-98. Politisch basiert „racial redress“ auf der vom ANC propagierten „transformation“ der Gesamtgesellschaft, welche auch den „Anspruch“ der Partei auf die Kontrolle des Staatsapparates beinhaltet. Vgl. hierzu Nicola De Jager, The ANC government, Co-optive Power and the Perpetuation of Party Dominance, in: Konrad Adenauer Stiftung South Africa (ed.): Challenges to Democracy by One-Party Dominance. A Comparative Assessment, Johannesburg 2006, S. 15-30.

Spitz / Chaskalson, oben Fn. 20, S. 228 ff.. 
posal consistent with democracy“. ${ }^{80}$ An dieser Stelle mag insbesondere die Überzeugung handlungsleitend gewesen sein, die Offenheit des politischen Wettbewerbs auch für die Zukunft zu sichern. Gerade angesichts der fragilen und zerklüfteten politischen Landschaft wurde durch den Verzicht auf Parteiverbots-Vorkehrungen die zukünftige Handhabe eines andere Akteure ausschließenden Vorgehens glaubhaft versperrt.

\section{II.3 Politischer Extremismus}

Im Hinblick auf ein mögliches anti-extremistisches Parteiverbot wurde in Kapitel 2 zumindest für die Liberalisierungs- und beginnende Demokratisierungsphase das Bestehen einer „objektiven Gefährdung“ festgestellt. Nur unter solchen Bedingungen der „objektiven Gefährdung“, darüber besteht Konsens, können Parteiverbote breite Legitimität beanspruchen. Insofern gehorchen sie einer Logik der Verhältnismäßigkeit. Die Ausgangslage der Verhandlungen wird von einem Befragten als ,stalemate were neither side could defeat the other" beschrieben. „Therefore neither side could impose on the other a provisison of excluding parties potentially useful or biased towards their side“. ${ }^{81}$ Auf die Gefährdung von Rechts- und Linksaußen reagierten die zentralen Akteure nicht mit Repression, sondern mit Inklusionsbemühungen. Eine militante Abgrenzung von den jeweiligen Radikalen des eigenen Lagers hätte zwischen 1990 und 1994 die Legitimität dieser Akteure beschädigt und die Gegner des friedlichen Übergangs in ihren Überzeugungen bestärkt, die Heribert Adam folgendermaßen umreißt: „,There were great doubts on the extreme right as well on the extreme left whether to join the political process at all and give up a romanticized 'armed struggle'. The racist AWB and their allies espoused the slogan 'bullets instead of ballots'. On the other side, many in the SACP and MK argued that you cannot win at the negotiating table what you have not won at the battlefield". 82

Mit dem Inkrafttreten der Interims-Verfassung und den ersten freien Wahlen 1994 änderte sich die „Gefährdungslage“ insofern, als die wichtigsten COSAG/FA Kräfte sich doch noch an den Wahlen beteiligten. Auf der weißen Rechten ging die „Freedom Front“ als legalistische Partei vor allem aus der gemäßigten Fraktion der AVF hervor. ${ }^{83}$ Auch Inkatha erklärte im letzten Moment ihre Beteiligung an den Wahlen und anerkannte damit die Legitimität des Verhandlungsprozesses. Ab 1994 kann damit die Einschätzung Klugs geteilt werden, dass die verbliebenen extremen Gruppen keine wirkliche Gefährdung der demokratischen Ordnung mehr darstellten, da sich erstens Militär und Administration in Krisenmomenten wie dem versuchten Staatsstreich in Bophuthatswana als loyal erwiesen hatten: ,The more extreme groups of all political persuasions were not considered a serious political or security threat to the new state. When these minor threats were compared to the

80

81

82

83

Zitiert in Ebrahim, oben Fn. 23, S. 530.

Fragebogen von Heribert Adam.

Ebd.

Spitz/Chaskalson, oben Fn. 20, S. 245. 
costs of undermining the broad rights-based stand of the new Constitution it would have been considered unduly costly to restrict these parties“. ${ }^{84}$ Zweitens muss das schwache Abschneiden fast aller Kritiker des neuen Systems in Betracht gezogen werden. So blieb die Freedom Front, der legalistische Zeig der Afrikaner-Rechten, mit wenig mehr als 2\% Wählerstimmen deutlich hinter den eigenen Hoffnungen zurück und musste die Pläne für einen „Volkstaat“ (oder „Afrikaner-Homeland“) zurückstellen. ${ }^{85}$ Während für die NPRegierung bis 1994 Parteiverbote ,not practical“ gewesen sind, war die ANC-Regierung nach 1994 ,simply not in any position to do this given the country's past and the experience of being banned themselves“". ${ }^{86}$ Ergänzt sei diese Überlegung allerdings durch ein machtstrategisches Argument: ,Since the ANC command a huge majority anyway, it never felt threatened by smaller parties as potential targets of banning“. ${ }^{87}$ Dank seiner Dominanz im Parteiensystem und im Parlament seit 1994 wäre der ANC derzeit die einzige politische Kraft, die ernsthaft die Einführung von Parteiverboten initiieren könnte. Durch Wahlen scheint die absolute Mehrheit des ANC zumindest mittelfristig nicht gefährdet ${ }^{88}$, so dass offene Repression gegen kleinere politische Gegner die Kritik an der „,dominant party rule“ stärken würde. ${ }^{89}$ Die Öffentlichkeit zeigt sich durchaus sensibel für Fehlentwicklungen und die Ein-Parteien-Dominanz wird als potenzielle Gefahr für die demokratische Verfassung wahrgenommen. ${ }^{90}$ Die einseitige Propagierung einer „,militant democracy“ von Seiten des ANC würde demnach sofort den Verdacht des Machtmissbrauchs aufkommen lassen. Diese Überlegung kann gleichermaßen auf die Akteurskonstellation während des Transformationsprozesses ausgeweitet werden. Da das Argument, Parteiverbote hätten gegen das Gebot der Verhältnismäßigkeit verstoßen, für den Zeitraum von der Liberalisierung des politischen Systems bis zu den ersten Wahlen kaum Geltung beanspruchen kann, sollen an dieser Stelle kurz die konkreten Akteursinteressen jenseits von Überzeugungen angesprochen werden. Sowohl der ANC als auch die NP hatten in den jeweiligen Lagern ein primäres Interesse an Aufrechterhaltung ihrer Reputation. Der „broad church approach“ des ANC zielte auf den Einbezug möglichst vieler Strömungen ab, natürlich auch vor dem Hintergrund der Sicherung der sich frühzeitig abzeichnenden dominanten Position in einem frei gewählten Parlament. Die NP, ebenso wie später ihre Nachfolgeorganisation New National

Fragebogen von Heinz Klug.

Der spätere Präsident Nelson Mandela hatte der Afrikaner-Rechte Verhandlungen über die Einrichtung eines „Volksstaats“ zugesagt, falls ein gutes Wahlergebnis dies nahegelegt hätte (Interview mit Hendrik Bussiek (Cape Town, 05.06.2008)).

Interview mit Robert Mattes (Cape Town, 03.06.2008).

Fragebogen von Heribert Adam.

Tom Lodge, The Future of South Africa's Party System, Journal of Democracy 17 (2006), S. 164.

Siehe Hermann Giliomee / James Myburg / Lawrence Schlemmer, Dominant Party Rule, Opposition Parties and Minorities in South Africa, in: Rogar Southall (ed.): Opposition and Democracy in South Africa, London 2001, S. 161-182.

90

De Jager, oben Fn. 78. 
Party, verfolgte eine ähnliche Strategie, in dem sie danach trachtete ihre Position als wichtigste ,nicht schwarze“ Partei aufrecht zu erhalten. ${ }^{91}$ Deswegen musste auch sie den Eindruck vermeiden, auf gerade erst überwundene und allgemein als illegitim empfundene repressive Instrumente zurück zu greifen.

\section{F. Ergebnis und vergleichende Perspektiven}

Dass die Südafrikanische Verfassung im Gegensatz zu den meisten anderen afrikanischen Transformationsverfassungen auf das Instrument des Parteiverbots verzichtet, erscheint in zweierlei Hinsicht bemerkenswert. Erstens, obwohl sich die Verfassungsgebung vor dem Hintergrund eines historischen und universell verurteilten Unrechtsregimes vollzog, reagierte sie nicht mit dem prospektiven Verbot politischer Akteure, die eine Wiederherstellung der diskreditierten Herrschaftsordnung anstrebten. Zweitens entstand die neue Demokratie aus gewalttätigen Konflikten und hält dennoch keine Instrumente vor, mit denen sie sich legaler antidemokratischer Mobilisierung erwehren kann. Diese auf den ersten Blick erstaunliche Sachlage hängt zunächst mit der vergangenheitspolitischen Deutung der Assoziationsverbote des Apartheidregimes zusammen. Während in anderen Kontexten Assoziationsverbote durchaus eine freiheitsverbürgende Funktion beanspruchen können, etwa die Parteiverbote in den anti-faschistischen Verfassungen Italiens und Portugals, konnte diese Sichtweise im südafrikanischen Transformationsdiskurs nicht Fuß fassen. Aufgrund der Identifikation solcher Verbote als Repressionsinstrumente grenzt auch die Verfassungsrechtslehre die Demokratiekonzeption der Südafrikanischen Verfassung deutlich vom Modell der „,militant democracy“ ab. ${ }^{92}$ Das Integrationsmodell der Verfassung betont dagegen viel stärker positive Werte, was insbesondere im allgemeinen Gleichheitsgebot in Art. 9 zum Ausdruck kommt. ${ }^{93}$ In struktureller Hinsicht geraten insbesondere die Faktoren des Transformationsprozesses in den Blick. Für die koordinierte Transition Südafrikas konnten drei zentrale Merkmale herausgearbeitet werden: die konstruktive Beteiligung der alten Eliten am Prozess, die Inklusivität des Prozesses und die Konsensorientierung der prospektiven neuen Eliten. Da mit der NP der maßgebliche Träger der Apartheid am Verhandlungstisch saß, musste die Abgrenzung vom Apartheidregime in der neuen Verfassung in allgemeinen Formulierungen verbleiben und konnte nicht auf konkrete Organisationen oder Parteien bezogen werden. Der angestrebten Inklusivität wäre durch ein Parteiverbot Schaden zugefügt worden, indem die transformations-kritischen Kräfte zusätzliche Legitimität erhalten hätten, besonders im Lichte der oben angestellten vergangenheitspolitischen

Während die Strategie für den ANC aufging, scheiterte die NNP auf ganzer Linie. Nach erheblichen Verlusten und einer gescheiterten Kooperation mit der DP löste sich die NNP nach der Wahl 2004 auf. Vor allem viele Parlamentarier der NNP traten dem ANC bei. Vgl. hierzu Roger Southall, South Africa, in: The KAS Democracy Report, Bonn 2007.

93 Siehe für viele Woolman, oben Fn. 38, S. 3.

Nikolaus Holzer, Integration durch Verfassung. Das Beispiel der Verfassungsgebung in der Republik Südafrika, Diss Mainz 1999. 
Überlegungen. Der ANC als zukünftige Dominanzpartei verfolgte zudem das Interesse, möglichst viele Strömungen einzubinden und natürlich auch einen möglichst wohlgeordneten Staat zu übernehmen. Deshalb wollte er alles vermeiden, was die Konflikte über die zukünftige Ordnung verschärft hätte. Zugespitzt: Im „Grand Design“ der Transformation und der Verfassungsgebung wären Parteiverbote letztlich ein systemfremdes Element.

Der Vorteil einer transformationsbezogenen Betrachtung liegt schließlich in der Eröffnung komparatistischer Anschlussperspektiven, die vom in sich scheinbar schlüssigen Einzelfall abstrahieren. In Andrew Aratos Transitionstypologie, die sich in ähnlicher Begrifflichkeit natürlich auch bei anderen Autoren findet ${ }^{94}$, konnte die südafrikanische Transition dem Typ der ,coordinated transition“ zugeordnet werden, die aufgrund der Inklusivität des Prozesses offenbar zu liberalen Verfassungsinstitutionen tendiert. In den anderen Transformationstypen, der von oben gelenkten Reform, bzw. ,managed transition“, und der Revolution oder des Regimezusammenbruchs, sollte die Einführung von Parteiverboten wahrscheinlicher sein. ${ }^{95}$ Beim ersten Typ deshalb, weil Einschränkungen politischer Freiheiten als Steuerungsinstrument in einem von oben kontrollierten Prozess angesehen werden können. Als Beispiele dafür können die Türkei gelten oder auch afrikanische Länder wie Nigeria und Tansania, in denen die schrittweise Demokratisierung von der Möglichkeit, Parteien zu verbieten und tatsächlichen Parteiverboten begleitet wird. Regimezusammenbrüche gehen oft mit einer vollständigen Diskreditierung der alten Ordnung einher, die mit deren Negierung in neuen demokratischen Verfassungen bestätigt wird. Beispiele sind das nach-faschistische Italien, Portugal und auch die Bundesrepublik der fünfziger Jahre, wo das Verbot der neo-nazistischen SRP explizit auch mit den verheerenden Erfahrungen des Dritten Reiches begründet wurde. Als Kontrastfall innerhalb der Kategorie der ,,coordinated transition“ könnte allerdings Polen gelten, wo der Systemwechsel ebenso am Runden Tisch zwischen den alten Eliten und ihren Herausforderern ausgehandelt wurde. ${ }^{96}$ Die Verfassung von 1997 sieht in Art. 13 aber ein Verbot nazistischer und kommunistischer Parteien vor. Diese Klausel kannten weder die revidierte Verfassung von 1989 noch die Übergangsverfassung von 1992 („Kleine Verfassung“). Vergleichbar werden die Fälle Südafrika und Polen dann, wenn der gestufte Verfassungsgebungsprozess, mit der „Kleinen Verfassung“ von 1992 und der „Interim Constitution“ von 1993 als erster sowie der „Großen Verfassung“ von 1997 und der „Final Constitution“ von 1996 als zweiter Stufe, als Parallele angesehen wird. Besonders bemerkenswert daran ist, dass sich in Polen im Zeitraum „zwischen“ den beiden Verfassungen offensichtlich eine Position herausbilden konnte, die gleichermaßen die Relevanz des restriktiven Demokratieschutzes betonte sowie dessen Stoßrichtung festlegte. In Südafrika hingegen besaß ,militant democracy“ nach Inkrafttreten der Übergangsverfassung keinerlei Relevanz in der öffentlichen und verfas-

Ein weiterer Typ, derjenige des „,self coup“ bzw. ,autogolpe“, bleibt an dieser Stelle außen vor. Vgl. hierzu Arato, oben Fn. 51, S. 411 ff..

96

Olivia Jazwinski, Unrechtsaufarbeitung nach einem Regimewechsel, Baden-Baden 2007. 
sungspolitischen Debatte. ${ }^{97}$ Dennoch lanciert, wäre sie eher als Angriff auf die junge Demokratie denn als ein Instrument zu deren Stützung interpretiert worden. ${ }^{98}$

Zumindest zwei Erklärungen bieten sich für diesen signifikanten Unterschied zwischen Südafrika und Polen an. Durch die Abgrenzung von Nazismus und Kommunismus wird in Polen eine symmetrische Externalisierung der Vergangenheit angestrebt, in dem die totalitären Herrschaftssysteme als von außen oktroyiert konzipiert werden. ${ }^{99}$ Diese Form der Externalisierung hätte indes für Südafrika nicht den intendierten ,befreienden“ Effekt besessen wie für Polen: Die Apartheid mitsamt Administratoren und Opfern, galt unzweifelhaft als genuin südafrikanisches Phänomen. Zum anderen kann die Stabilität des politischen und insbesondere des Parteiensystems angeführt werden. Das polnische Parteiensystem der neunziger Jahre (und auch darüber hinaus) zeichnet sich im Gegensatz zum südafrikanischen durch ein hohes $\mathrm{Ma} 3$ an Fluidität aus. ${ }^{100}$ Während Südafrika seit 1994, bislang bedingt durch die Prädominanz des ANC, stabile Regierungen hervorbrachte, waren die polnischen Kabinette der Post-Transitionsphase durch häufige Wechsel der beteiligten Partner gekennzeichnet

Hier kommt sowohl Sartoris Argument von Parteiverboten als stabilitätsverbürgenden Instrumenten als auch das Motiv der Rückfallsperre ins Spiel. Das Verbot „extremistischer“ Parteien (denn Art. 13 der Polnischen Verfassung bezieht sich nicht nur historisch auf nazistische und kommunistische Parteien, sondern auch konkret auf Praktiken des Rassenund Nationalitätenhasses sowie der Gewalt), ist also gleichsam zu verstehen als Schutz gegen eine Beschädigung der neuen Demokratie oder sogar gegen einen „Rückfall“ in vordemokratische Verhältnisse. Angesichts der Instabilität des polnischen Parteiensystems Mitte der neunziger Jahre war diese Gefahr dort möglicherweise virulenter als in Südafrika.

100

So der übergreifende Tenor der in Südafrika interviewten Experten.

Zudem stand die „Final Constitution“ unter dem Prüfungsvorbehalt des Verfassungsgerichtes. Gemäß der dominierenden Verfassungsinterpretation hätte dieses Parteiverbotsklauseln nach deutschem oder auch polnischem Muster wohl zurückweisen müssen.

Zum polnischen Streit um den Umgang mit der kommunistischen Geschichte vgl. Krzysztof Ruchniewicz, Der Umgang mit der Vergangenheit in postkommunistischen Gesellschaften. Polen nach 1989, in: Alfons Kenkmann / Hasko Zimmer (Hrsg.), Nach Kriegen und Diktaturen. Umgang mit Vergangenheit als internationales Problem - Bilanzen und Perspektiven für das 21. Jahrhundert, 2006, S. 69-80.

James Toole, Government Formation and Party System Stabilization in East Central Europe, Party Politics 6 (2000), S. 441-461. 